\title{
On the speed of a cookie random walk
}

\author{
Anne-Laure Basdevant and Arvind Singh * \\ University Paris VI
}

\begin{abstract}
We consider the model of the one-dimensional cookie random walk when the initial cookie distribution is spatially uniform and the number of cookies per site is finite. We give a criterion to decide whether the limiting speed of the walk is non-zero. In particular, we show that a positive speed may be obtained for just 3 cookies per site. We also prove a result on the continuity of the speed with respect to the initial cookie distribution.
\end{abstract}

Keywords. Law of large numbers, cookie or multi-excited random walk, branching process with migration

A.M.S. Classification. 60K35, 60J80, 60F15

e-mail. anne-laure.basdevant@ens.fr, arvind.singh@ens.fr

\section{Introduction}

We consider the model of the multi-excited random walk, also called cookie random walk, introduced by Zerner in [10] as a generalization of the model of the excited random walk described by Benjamini and Wilson in [2] (see also Davis [3] for a continuous time analogue). The aim of this paper is to study under which conditions the speed of a cookie random walk is strictly positive. In dimension $d \geq 2$, this problem was solved by Kozma [6. 7] who proved that the speed is always non-zero. In the one-dimensional case, the speed can either be zero or strictly positive. We give here a necessary and sufficient condition to determine if the walk's speed is strictly positive when the initial cookie environment is deterministic, spatially uniform and with a finite number of cookies per site. Let us start with an informal definition of such a process:

Let us put $M \geq 1$ "cookies" at each site of $\mathbb{Z}$ and let us pick $p_{1}, p_{2}, \ldots, p_{M} \in\left[\frac{1}{2}, 1\right)$. We say that $p_{i}$ represents the "strength" of the $i^{\text {th }}$ cookie at any given site. Then, a cookie random walk $X=\left(X_{n}\right)_{n \geq 0}$ is simply a nearest neighbour random walk, eating the cookies it finds along its path by behaving in the following way:

*Address for both authors: Laboratoire de Probabilités et Modèles Aléatoires, Université Pierre et Marie Curie, 175 rue du Chevaleret, 75013 Paris, France. 
- If $X_{n}=x$ and there is no remaining cookie at site $x$, then $X$ jumps at time $n+1$ to $x+1$ or $x-1$ with equal probability $\frac{1}{2}$.

- If $X_{n}=x$ and there remain the cookies with strengths $p_{j}, p_{j+1}, \ldots, p_{M}$ at this site, then $X$ eats the cookie with attached strength $p_{j}$ (which therefore disappears from this site) and then jumps at time $n+1$ to $x+1$ with probability $p_{j}$ and to $x-1$ with probability $1-p_{j}$.

This model is a particular case of self-interacting random walk: the position of $X$ at time $n+1$ depends not only of its position at time $n$ but also on the number of previous visits to its present site. Therefore, $X$ is not a Markov process.

Let us now give a formal description of the general model. We define the set of cookie environments by $\Omega=\left[\frac{1}{2}, 1\right]^{\mathbb{N}^{*} \times \mathbb{Z}}$. Thus, a cookie environment is of the form $\omega=$ $(\omega(i, x))_{i \geq 1, x \in \mathbb{Z}}$ where $\omega(i, x)$ represents the strength of the $i^{\text {th }}$ cookie at site $x$. Given $x \in \mathbb{Z}$ and $\omega \in \Omega$, a cookie random walk starting from $x$ in the cookie environment $\omega$ is a process $\left(X_{n}\right)_{n \geq 0}$ on some probability space $\left(\Omega, \mathcal{F}, \mathbf{P}_{\omega, x}\right)$ such that:

$$
\left\{\begin{array}{l}
\mathbf{P}_{\omega, x}\left\{X_{0}=z\right\}=1, \\
\mathbf{P}_{\omega, x}\left\{\left|X_{n+1}-X_{n}\right|=1\right\}=1, \\
\mathbf{P}_{\omega, x}\left\{X_{n+1}=X_{n}+1 \mid X_{1}, \ldots, X_{n}\right\}=\omega\left(j, X_{n}\right) \text { where } j=\sharp\left\{0 \leq i \leq n, X_{i}=X_{n}\right\} .
\end{array}\right.
$$

In this paper, we restrict our attention to the set of environments $\Omega_{M}^{u} \subset \Omega$ which are spatially uniform with at most $M \geq 1$ cookies per site:

$$
\omega \in \Omega_{M}^{u} \Longleftrightarrow\left\{\begin{array}{l}
\text { for all } x \in \mathbb{Z} \text { and all } i \geq 1 \omega(i, x)=\omega(i, 0) \\
\text { for all } i>M \omega(i, 0)=\frac{1}{2} \\
\text { for all } i \geq 1 \omega(i, 0)<1
\end{array}\right.
$$

The last condition $\omega(i, 0)<1$ is introduced only to exclude some possible degenerated cases but can be relaxed (see Remark 2.4). A cookie environment $\omega \in \Omega_{M}^{u}$ may be represented by $(M, \bar{p})$ where

$$
\bar{p}=\left(p_{1}, \ldots, p_{M}\right)=(\omega(1,0), \ldots, \omega(M, 0)) .
$$

In this case, we shall say that the associated cookie random walk is an $(M, \bar{p})$-cookie random walk and we will use the notation $\mathbf{P}_{(M, \bar{p})}$ instead of $\mathbf{P}_{\omega}$.

The question of the recurrence or transience of a cookie random walk was solved by Zerner in [10] for general cookie environments (even in the case where the initial cookie environment may itself be random). In particular, he proved that, if $X$ is an $(M, \bar{p})$ cookie random walk, there is a phase transition according to the value of

$$
\alpha=\alpha(M, \bar{p}) \stackrel{\text { def }}{=} \sum_{i=1}^{M}\left(2 p_{i}-1\right)-1 .
$$

- If $\alpha \leq 0$ then the walk is recurrent i.e. $\lim \sup X_{n}=-\liminf X_{n}=+\infty$ a.s.

- If $\alpha>0$ then $X$ is transient toward $+\infty$ i.e $\lim X_{n}=+\infty$ a.s. 
In particular, for $M=1$, the cookie random walk is always recurrent for any choice of $\bar{p}$. However, as soon as $M \geq 2$, the cookie random walk can either be transient or recurrent depending on $\bar{p}$. Zerner [10] also proved that the speed of a $(M, \bar{p})$-cookie random walk $X$ is always well defined (but may or not be zero). Precisely,

- there exists a constant $v(M, \bar{p}) \geq 0$ such that

$$
\frac{X_{n}}{n} \underset{n \rightarrow \infty}{\longrightarrow} v(M, \bar{p}) \quad \mathbf{P}_{(M, \bar{p}) \text {-almost surely. }}
$$

- The speed is monotonic in $\bar{p}$ : if $\bar{p}=\left(p_{1}, \ldots, p_{M}\right)$ and $\bar{q}=\left(q_{1}, \ldots, q_{M}\right)$ are two cookie environments such that $p_{i} \leq q_{i}$ for all $i$, then $v(M, \bar{p}) \leq v(M, \bar{q})$.

- The speed of a $(2, \bar{p})$-cookie random walk is always 0 .

The question of whether one can construct a $(M, \bar{p})$-cookie random walk with strictly positive speed was affirmatively answered by Mountford, Pimentel and Valle [8] who considered the case where all the cookies have the same strength $p \in\left[\frac{1}{2}, 1\right)$ i.e. the cookie vector $\bar{p}$ has the form $[p]_{M} \stackrel{\text { def }}{=}(p, \ldots, p)$. They showed that:

- For any $p \in\left(\frac{1}{2}, 1\right)$, there exists an $M_{0}$ such that for all $M>M_{0}$ the speed of the $\left(M,[p]_{M}\right)$-cookie random walk is strictly positive.

- If $M(2 p-1)<2$, then the speed of the $\left(M,[p]_{M}\right)$-cookie random walk is zero.

They also conjectured that when $M(2 p-1)>2$, the speed should be non-zero. The aim of this paper is to prove that such is indeed the case.

Theorem 1.1. Let $X$ denote a $(M, \bar{p})$-cookie random walk, then

$$
\lim _{n \rightarrow \infty} \frac{X_{n}}{n}=v(M, \bar{p})>0 \quad \Longleftrightarrow \quad \alpha(M, \bar{p})>1
$$

where $\alpha(M, \bar{p})$ is given by (11).

In particular, we see that a non-zero speed may be achieved for as few as 3 cookies per site. Comparing this result with the transience/recurrence criteria, we have a second order phase transition at the critical value $\alpha=1$. In fact, it shall be proved in a forthcoming paper that, for $0<\alpha<1$, the rate of transience of $X_{n}$ is of order $n^{\frac{\alpha+1}{2}}$.

One would certainly like an explicit calculation of the limiting velocity in term of the cookie environment $(M, \bar{p})$ but this seems a challenging problem (one can still look at the end of Section 3 where we give an implicit formula for the speed). However, one can prove that the speed is continuous in $\bar{p}$ and has a positive right derivative at all its critical points:

Theorem 1.2. - For each $M$, the speed $v(M, \bar{p})$ is a continuous function of $\bar{p}$ in $\Omega_{M}^{u}$.

- For any environment $\left(M, \bar{p}_{c}\right)$ with $\alpha\left(M, \bar{p}_{c}\right)=1$, there exists a constant $C>0$ (depending on $\left(M, \bar{p}_{c}\right)$ ) such that

$$
\lim _{\substack{\bar{p} \rightarrow \bar{p}_{c} \\ \bar{p} \in \Omega_{M}^{u} \\ \alpha(\bar{p})>1}} \frac{v(M, \bar{p})}{\alpha(M, \bar{p})-1}=C .
$$


In particular, for $M \geq 3$, the (unique) critical value for an $\left(M,[p]_{M}\right)$-cookie random walk is $p_{c}=\frac{1}{M}+\frac{1}{2}$ and the function $v(p)$ is continuous, non-decreasing, zero for $p \leq p_{c}$, and admits a finite strictly positive right derivative at $p_{c}$.

The remainder of this paper is organized as follow. In the next section, we construct a Markov process associated with the hitting time of the cookie random walk. The method is similar to that used by Kesten, Kozlov and Spitzer [5] for the determination of the rates of transience of a random walk in a one-dimensional random environment. It turns out that, in our setting, the resulting process is a branching process with random migration. The study of this process and of its stationary distribution is done in Section 3. This enables us to complete the proof of Theorem 1.1. Finally, the last section is dedicated to the proof of Theorem 1.2 .

\section{An associated branching process with migration}

In the remainder of this paper, $X=\left(X_{n}\right)_{n \geq 0}$ will denote a $(M, \bar{p})$-cookie random walk. Since the speed of a recurrent cookie random walk is zero, we will also assume that we are in the transient regime i.e.

$$
\alpha(M, \bar{p})=\sum_{i=1}^{M}\left(2 p_{i}-1\right)-1>0 .
$$

For the sake of brevity, we simply write $\mathbf{P}_{x}$ for $\mathbf{P}_{(M, \bar{p}), x}$ and $\mathbf{P}$ instead of $\mathbf{P}_{0}$ (the process starting from 0$)$. Let $T_{n}$ stand for the hitting time of level $n \geq 0$ by $X$ :

$$
T_{n}=\inf \left(k \geq 0, X_{k}=n\right) .
$$

For $0 \leq k \leq n$, let $U_{i}^{n}$ denote the number of jumps of the cookie random walk from site $i$ to site $i-1$ before reaching level $n$

$$
U_{i}^{n}=\sharp\left\{0 \leq k<T_{n}, X_{k}=i \text { and } X_{k+1}=i-1\right\} .
$$

Let also $K_{n}$ stand for the total time spent by $X$ in the negative half-line up to time $T_{n}$

$$
K_{n}=\sharp\left\{0 \leq k \leq T_{n}, X_{k}<0\right\} .
$$

A simple combinatorial argument readily yields

$$
T_{n}=K_{n}-U_{0}^{n}+n+2 \sum_{k=0}^{n} U_{k}^{n} .
$$

Notice that, as $n$ tends to infinity, the random variable $K_{n}$ increases almost surely toward $K_{\infty}$, the total time spent by the cookie random walk in the negative half line. Similarly, $U_{0}^{n}$ increases toward $U_{0}^{\infty}$ the total number of jumps from 0 to -1 . Since $X$ is transient, $K_{\infty}+U_{0}^{\infty}$ is almost-surely finite and therefore

$$
T_{n} \underset{n \rightarrow \infty}{\sim} n+2 \sum_{k=0}^{n} U_{k}^{n} .
$$


Let us now prove that for each $n$, the reverse process $\left(U_{n}^{n}, U_{n-1}^{n}, \ldots, U_{1}^{n}, U_{0}^{n}\right)$ has the same law as the $n$ first steps of some branching process $Z$ with random migration. We first need to introduce some notations. Let $\left(B_{i}\right)_{i \geq 1}$ denote a sequence of independent Bernoulli random variable under $\mathbf{P}$ with distribution:

$$
\mathbf{P}\left\{B_{i}=1\right\}=1-\mathbf{P}\left\{B_{i}=0\right\}= \begin{cases}p_{i} & \text { if } i \leq M, \\ \frac{1}{2} & \text { if } i>M .\end{cases}
$$

For $j \in \mathbb{N}$, define

$$
k_{j}=\min \left(k \geq 1, \sharp\left\{1 \leq i \leq k, B_{i}=1\right\}=j+1\right)
$$

and

$$
A_{j}=\sharp\left\{1 \leq i \leq k_{j}, B_{i}=0\right\}=k_{j}-j-1 .
$$

We have the following easy lemma:

Lemma 2.1. - For any $i, j \geq 0$, we have $\mathbf{P}\left\{A_{j}=i\right\}>0$.

- For all $j \geq M$, we have

$$
A_{j} \stackrel{\text { law }}{=} A_{M-1}+\xi_{1}+\ldots+\xi_{j-M+1}
$$

where $\left(\xi_{i}\right)_{i \geq 0}$ is a sequence of i.i.d. geometrical random variable with parameter $\frac{1}{2}$ independent of $A_{M-1}$.

Proof. The first part of the lemma is a direct consequence of the assumption that $\bar{p}$ is such that $p_{k}<1$ for all $k$. To prove the second part, we simply notice that $k_{M-1} \geq M$ so that for $j \geq M$, the random variable $A_{j}-A_{M-1}$ has the same law as the random variable

$$
\min \left(k \geq 1, \sharp\left\{1 \leq i \leq k, \widetilde{B}_{i}=1\right\}=j+1-M\right)-j-1+M
$$

where $\left(\widetilde{B}_{i}\right)_{i \geq 0}$ is a sequence of i.i.d. random variables independent of $A_{M-1}$ and with common Bernoulli distribution $\mathbf{P}\left\{\widetilde{B}_{i}=0\right\}=\mathbf{P}\left\{\widetilde{B}_{i}=1\right\}=\frac{1}{2}$. It is clear that (7) has the same law as $\xi_{1}+\ldots+\xi_{j-M+1}$.

By possibly extending the probability space, we now construct a process $Z=\left(Z_{n}, n \geq\right.$ 0 ) and a family of probability $\left(\mathbb{P}_{z}\right)_{z \geq 0}$ such that, under $\mathbb{P}_{z}$, the process $Z$ is a Markov chain starting from $z$, with transition probability:

$$
\left\{\begin{array}{l}
\mathbb{P}_{z}\left\{Z_{0}=z\right\}=1 \\
\mathbb{P}_{z}\left\{Z_{n+1}=k \mid Z_{n}=j\right\}=\mathbf{P}\left\{A_{j}=k\right\}
\end{array}\right.
$$

Since the family of probabilities $\left(\mathbb{P}_{z}\right)$ depends on the law of the cookie environment $(M, \bar{p})$, we should rigourously write $\mathbb{P}_{(M, \bar{p}), z}$ instead of $\mathbb{P}_{z}$. However, when there is no possible confusion we will keep using the abbreviated notation. Furthermore, we will simply write $\mathbb{P}$ for $\mathbb{P}_{0}$ and $\mathbb{E}$ will stand for the expectation with respect to $\mathbb{P}$.

Let us now notice that, in view of the previous lemma, $Z_{n}$ under $\mathbb{P}_{z}$ may be interpreted as the number of particles alive at time $n$ of a branching process with random migration starting from $z$, that is a branching process which allows immigration and emigration (see Vatutin and Zubkov [9] for a survey on these processes). Indeed: 
- If $Z_{n}=j \geq M-1$, then according to Lemma 2.1, $Z_{n+1}$ has the same law as $\sum_{k=1}^{j-M+1} \xi_{k}+A_{M-1}$, i.e. $M-1$ particles emigrate and the remaining particles reproduce according to a geometrical law with parameter $\frac{1}{2}$ and there is also an immigration of $A_{M-1}$ new particles.

- If $Z_{n}=j \in\{0, \ldots, M-2\}$ then $Z_{n+1}$ has the same law as $A_{j}$ i.e. all the $j$ particles emigrate and $A_{j}$ new particles immigrate.

We can now state the main result of this section:

Proposition 2.2. For each $n \in \mathbb{N},\left(U_{n}^{n}, U_{n-1}^{n}, \ldots, U_{0}^{n}\right)$ under $\mathbf{P}$ has the same law as $\left(Z_{0}, Z_{1}, \ldots, Z_{n}\right)$ under $\mathbb{P}$.

Proof. The argument is similar to the one given by Kesten et al. in [5]. Recall that $U_{i}^{n}$ represents the numbers of jumps of the cookie random walk $X$ from $i$ to $i-1$ before reaching $n$. Then, conditionally on $\left(U_{n}^{n}, U_{n-1}^{n}, \ldots, U_{i+1}^{n}\right)$, the number of jumps $U_{i}^{n}$ from $i$ to $i-1$ depends only on the number of jumps from $i+1$ to $i$, that is, depends only of $U_{i+1}^{n}$. This shows that $\left(U_{n}^{n}, U_{n-1}^{n}, \ldots, U_{0}^{n}\right)$ is indeed a Markov process.

By definition, $Z_{0}=0 \mathbb{P}$-a.s. and $U_{n}^{n}=0 \mathbf{P}$-a.s. It remains to compute $\mathbf{P}\left\{U_{i}^{n}=\right.$ $\left.k \mid U_{i+1}^{n}=j\right\}$. Note that the number of jumps from $i$ to $i-1$ before reaching level $n$ is equal to the number of jumps from $i$ to $i-1$ before reaching $i+1$ for the first time plus the sum of the number of jumps from $i$ to $i-1$ between two consecutive jumps from $i+1$ to $i$ which occur before reaching level $n$. Thus, conditionally on $\left\{U_{i+1}^{n}=j\right\}$, the random variable $U_{i}^{n}$ has the same law as the number of failures (i.e. $\left.B_{k}=0\right)$ in the Bernoulli sequence $\left(B_{1}, B_{2}, B_{3}, \ldots\right)$ defined by (5) before having exactly $j+1$ successes. This is precisely the definition of $A_{j}$ and therefore $\mathbf{P}\left\{U_{i}^{n}=k \mid U_{i+1}^{n}=j\right\}=\mathbb{P}_{j}\left\{Z_{1}=k\right\}$.

Since $U_{0}^{n}$ is the number of jumps from 0 to -1 of the cookie random walk $X$ before reaching level $n$ and since we assumed that the cookie random walk $X$ is transient, $U_{0}^{n}$ increases almost surely toward the total number $U_{0}^{\infty}$ of jumps of $X$ from 0 to -1 . In view of the previous proposition, this implies that under $\mathbb{P}, Z_{n}$ converges in law toward a random variable which we denote by $Z_{\infty}$.

Let us also note that $Z$ is a irreducible Markov chain (this is a consequence of part 1 of Lemma 2.1). Since $Z$ converges in law toward a limiting distribution, this shows that $Z$ is in fact a positive recurrent Markov chain. In particular, $Z_{n}$ converges in law toward $Z_{\infty}$ independently of its starting point (i.e. the law of $Z_{\infty}$ is the same under any $\mathbb{P}_{x}$ ) and the law of $Z_{\infty}$ is also the unique invariant probability for $Z$.

Corollary 2.3. Recall that $v(M, \bar{p})$ denotes the limiting speed of the cookie random walk $X$. We have

$$
\left.v(M, \bar{p})=\frac{1}{1+2 \mathbb{E}\left[Z_{\infty}\right]} \quad \text { (with the convention } 0=\frac{1}{+\infty}\right) .
$$

In particular, the speed of an $(M, \bar{p})$-cookie random walk is non zero i.i.f. the limiting random variable $Z_{\infty}$ of its associated process $Z$ has a finite expectation.

Proof. Since $X$ is transient, we have the well known equivalence valid for $v \in[0, \infty]$ :

$$
\frac{X_{n}}{n} \underset{n \rightarrow \infty}{\longrightarrow} v \quad \text { P-a.s. } \quad \Longleftrightarrow \quad \frac{T_{n}}{n} \underset{n \rightarrow \infty}{\longrightarrow} \frac{1}{v} \quad \text { P-a.s. }
$$


On the one hand, this equivalence and (44) yield

$$
\frac{1}{n} \sum_{k=0}^{n} U_{k}^{n} \underset{n \rightarrow \infty}{\longrightarrow} \frac{1}{2 v(M, \bar{p})}-\frac{1}{2} \quad \text { P-a.s. }
$$

On the other hand, making use of an ergodic theorem for the positive recurrent Markov chains $Z$ with stationary limiting distribution $Z_{\infty}$, we find that

$$
\frac{1}{n} \sum_{i=1}^{n} Z_{k} \underset{n \rightarrow \infty}{\rightarrow} \mathbb{E}\left[Z_{\infty}\right] \quad \mathbb{P} \text {-a.s. }
$$

(this result is valid even if $\mathbb{E}\left[Z_{\infty}\right]=\infty$ ). Proposition 2.2 implies that the limits in (9) and (10) are the same. This completes the proof of the corollary.

Remark 2.4. We assumed in the definition of an $(M, \bar{p})$ cookie environment that

$$
p_{i} \neq 1 \quad \text { for all } 1 \leq i \leq M \text {. }
$$

This hypothesis is intended only to ensure that $Z$ starting from 0 is not almost surely bounded (for instance, if $p_{1}=1$ then 0 is a absorbing state for $Z$ ). More generally, one may check from the definition of the random variables $A_{j}$ that $Z$ starting from 0 is almost surely unbounded i.i.f.

$$
\sharp\left\{1 \leq j \leq i, p_{j}=1\right\} \leq \frac{i}{2} \quad \text { for all } 1 \leq i \leq M .
$$

When this condition fails, $Z$ starting from 0 is almost surely bounded by $M-1$, thus $\mathbb{E}\left[Z_{\infty}\right]<\infty$ and the speed of the associated cookie random walk is strictly positive. Otherwise, when (11) is fulfilled, $Z$ ultimately hits any level $x \in \mathbb{N}$ with probability 1 and the proof of Theorem 1.1 remains valid.

\section{Study of $Z_{\infty}$.}

We proved in the previous section that the strict positivity of the speed of the cookie random walk $X$ is equivalent to the existence of a finite first moment for the limiting distribution of its associated Markov chain $Z$. We shall now show that, for any cookie environment $(M, \bar{p})$ (with $\alpha(M, \bar{p})>0$ ), we have

$$
\mathbb{E}\left[Z_{\infty}\right] \stackrel{\text { def }}{=} \mathbb{E}_{(M, \bar{p})}\left[Z_{\infty}\right]<\infty \quad \Longleftrightarrow \quad \alpha(M, \bar{p})>1 .
$$

This will complete the proof of Theorem 1.1. We start by proving that $Z_{\infty}$ cannot have moments of any order.

Proposition 3.1. We have

$$
\mathbb{E}\left[Z_{\infty}^{M-1}\right]=+\infty
$$


Proof. Let us introduce the first return time to 0 for $Z$ :

$$
\sigma=\inf \left(n \geq 1, Z_{n}=0\right) .
$$

Since $Z$ is a positive recurrent Markov chain, we have $1 \leq \mathbb{E}_{0}[\sigma]<\infty$ and the invariant probability measure is given for any $y \in \mathbb{N}$ by

$$
\mathbb{P}\left\{Z_{\infty}=y\right\}=\frac{\mathbb{E}_{0}\left[\sum_{k=0}^{\sigma-1} \mathbb{1}_{Z_{k}=y}\right]}{\mathbb{E}_{0}[\sigma]} .
$$

A monotone convergence argument yields

$$
\mathbb{E}_{0}\left[\sum_{k=0}^{\sigma-1} Z_{k}^{M-1}\right]=\mathbb{E}_{0}[\sigma] \mathbb{E}\left[Z_{\infty}^{M-1}\right]
$$

(where both side of this equality may be infinite). We can find $n_{0} \in \mathbb{N}^{*}$ such that $\mathbb{P}_{0}\left\{Z_{n_{0}}=M, n_{0}<\sigma\right\}>0$ (in fact, since we assume that $p_{i}<1$ for all $i$, we can choose $\left.n_{0}=1\right)$. Therefore, making use of the Markov property of $Z$, we find that

$$
\begin{aligned}
\mathbb{E}_{0}\left[\sum_{k=0}^{\sigma-1} Z_{k}^{M-1}\right] & \geq \mathbb{P}_{0}\left\{Z_{n_{0}}=M, n_{0}<\sigma\right\} \mathbb{E}_{M}\left[\sum_{k=0}^{\sigma-1} Z_{k}^{M-1}\right] \\
& =\mathbb{P}_{0}\left\{Z_{n_{0}}=M, n_{0}<\sigma\right\} \sum_{k=0}^{\infty} \mathbb{E}_{M}\left[Z_{k \wedge \sigma}^{M-1}\right] .
\end{aligned}
$$

In view of (12) and (13), we just need to prove that

$$
\sum_{k=0}^{\infty} \mathbb{E}_{M}\left[Z_{k \wedge \sigma}^{M-1}\right]=\infty
$$

We now use a coupling argument. Let us define a new Markov chain $\widetilde{Z}$ such that, under $\mathbb{P}_{z}$, the process evolves in the following way

- $\widetilde{Z}_{0}=z$,

- if $\widetilde{Z}_{n}=k \in\{0,1, \ldots, M-1\}$ then $\widetilde{Z}_{n+1}=0$,

- if $\widetilde{Z}_{n}=k>M-1$ then $\widetilde{Z}_{n+1}$ has the same law as $\sum_{i=1}^{k-(M-1)} \xi_{i}$ where $\left(\xi_{i}\right)_{i \geq 1}$ is a sequence of i.i.d. geometrical random variables with parameter $\frac{1}{2}$.

Thus, $\widetilde{Z}$ is a branching process with emigration: at each time $n$, there are $\min \left(\widetilde{Z}_{n}, M-1\right)$ particles which emigrate the system and the remaining particles reproduce according to a geometrical law of parameter $\frac{1}{2}$.

Recall that $Z$ is a branching process with migration, where at most $M-1$ particles emigrate at each unit of time, and has the same offspring reproduction law as $\widetilde{Z}$. Therefore, for any $z \geq 0$, under $\mathbb{P}_{z}$, the process $\widetilde{Z}$ is stochastically dominated by $Z$. Since 0 is an absorbing state for $\widetilde{Z}$, this implies that, for all $n \geq 0$ and all $z \geq 0$,

$$
\mathbb{E}_{z}\left[\widetilde{Z}_{n}^{M-1}\right] \leq \mathbb{E}_{z}\left[Z_{n \wedge \sigma}^{M-1}\right]
$$


Our process $\widetilde{Z}$ belongs to the class of processes studied by Kaverin [4]. Moreover, all the hypotheses of Theorem 1 of [4] are clearly fulfilled (in the notation of 4], we have here $\lambda=\theta=M-1$ and $B=1)$. Therefore, for any $z \geq M$, there exists a constant $c>0$ (depending on $z$ ) such that

$$
\mathbb{E}_{z}\left[\widetilde{Z}_{n}^{M-1}\right] \underset{n \rightarrow \infty}{\sim} \frac{c}{n}
$$

The combination of (15) and (16) yield (14).

Remark 3.2. In view of the last proposition and Corollary 2.3, we recover the fact that for $M=2$, the speed of the cookie random walk is always zero.

In order to study more precisely the distribution of $Z_{\infty}$, we will need the following lemma

Lemma 3.3. We have

$$
\mathbf{E}\left[A_{M-1}\right]=2 \sum_{i=1}^{M}\left(1-p_{i}\right)
$$

Proof. Recall that $\left(B_{i}\right)_{i \geq 1}$ denotes a sequence of independent Bernoulli random variables with distribution given by (5). Recall also that

$$
\begin{aligned}
& k_{M-1}=\min \left(k \geq 1, \sharp\left\{1 \leq i \leq k, B_{i}=1\right\}=M\right), \\
& A_{M-1}=k_{M-1}-M .
\end{aligned}
$$

In particular, for any $j \in \mathbb{N}^{*}$

$$
\begin{aligned}
\mathbf{P}\left\{A_{M-1}=j\right\} & =\mathbf{P}\left\{k_{M-1}=M+j\right\} \\
& =\mathbf{P}\left\{\sharp\left\{1 \leq i \leq M+j-1, B_{i}=1\right\}=M-1\right\} \mathbf{P}\left\{B_{M+j}=1\right\} .
\end{aligned}
$$

Hence,

$$
\begin{aligned}
& \mathbf{P}\left\{A_{M-1}=j\right\} \\
& =\frac{1}{2} \sum_{l=1}^{M} \mathbf{P}\left\{\sharp\left\{1 \leq i \leq M, B_{i}=1\right\}=M-l\right\} \mathbf{P}\left\{\sharp\left\{M+1 \leq i \leq M+j-1, B_{i}=1\right\}=l-1\right\} \\
& =\sum_{l=1}^{M \wedge j} \mathbf{P}\left\{\sharp\left\{1 \leq i \leq M, B_{i}=1\right\}=M-l\right\} C_{j-1}^{l-1}\left(\frac{1}{2}\right)^{j} .
\end{aligned}
$$

Let $L=\sharp\left\{1 \leq i \leq M, B_{i}=0\right\}$, therefore

$$
\mathbf{P}\left\{A_{M-1}=j\right\}=\sum_{l=1}^{M \wedge j} \mathbf{P}\{L=l\} C_{j-1}^{l-1}\left(\frac{1}{2}\right)^{j} \quad \text { for } j \in \mathbb{N}^{*} .
$$


Making use of the relation $j C_{j-1}^{l-1}=l C_{j}^{l}$, we get

$$
\begin{aligned}
\mathbf{E}\left[A_{M-1}\right] & =\sum_{j=1}^{\infty} \sum_{l=1}^{M \wedge j} \mathbf{P}\{L=l\}\left(\frac{1}{2}\right)^{j} l C_{j}^{l} \\
& =\sum_{l=0}^{M} l \mathbf{P}\{L=l\} \sum_{j=l}^{\infty}\left(\frac{1}{2}\right)^{j} C_{j}^{l} \\
& =2 \sum_{l=0}^{M} l \mathbf{P}\{L=l\} \\
& =2 \mathbf{E}[L] .
\end{aligned}
$$

We now compute $\mathbf{E}[L]$ by induction on the number of cookies.

$$
\mathbf{P}\{L=l\}=\sum_{1 \leq i_{1}<i_{2}<\ldots<i_{l} \leq M} \prod_{j=1}^{l}\left(1-p_{i_{j}}\right) \prod_{j \notin\left\{i_{1}, \ldots, i_{l}\right\}} p_{j} \quad \text { for } 0 \leq l \leq M .
$$

Decomposing the last sum according to whether $i_{1}=1$ or $i_{1} \neq 1$, we obtain

$\mathbf{E}[L]$

$$
\begin{aligned}
& =\sum_{l=1}^{M} l \sum_{2 \leq i_{2}<\ldots<i_{l} \leq M}\left(1-p_{1}\right) \prod_{j=2}^{l}\left(1-p_{i_{j}}\right) \prod_{j \notin\left\{1, i_{2}, \ldots, i_{l}\right\}} p_{j}+\sum_{l=0}^{M-1} l \sum_{2 \leq i_{1}<\ldots<i_{l} \leq M} \prod_{j=1}^{l}\left(1-p_{i_{j}}\right) p_{1} \prod_{j \notin\left\{1, i_{1}, \ldots, i_{l}\right\}} p_{j} \\
& =\sum_{l=0}^{l}\left(l-\left(1-p_{1}\right)\right) \sum_{2 \leq i_{1}<\ldots<i_{l} \leq M} \prod_{j=1}^{l}\left(1-p_{i_{j}}\right) \prod_{j \notin\left\{1, i_{1}, \ldots, i_{l}\right\}} p_{j} \\
& =\sum_{l=0}^{M-1}\left(l-\left(1-p_{1}\right)\right) \mathbf{P}\{\tilde{L}=l\} \\
& =\mathbf{E}[\tilde{L}]+1-p_{1},
\end{aligned}
$$

where $\tilde{L}=\sharp\left\{2 \leq i \leq M, B_{i}=0\right\}$. Finally, we conclude by induction that

$$
\mathbf{E}[L]=\sum_{i=1}^{M}\left(1-p_{i}\right)
$$

We now study the law of the limiting distribution $Z_{\infty}$ of the Markov chain $Z$. This is done via the study of its probability generating function (p.g.f.)

$$
G(s)=\mathbb{E}\left[s^{Z_{\infty}}\right] \quad \text { for } s \in[0,1] .
$$

Lemma 3.4. The p.g.f. $G$ of $Z_{\infty}$ is the unique p.g.f. solution of the following equation

$$
1-G\left(\frac{1}{2-s}\right)=a(s)(1-G(s))+b(s) \quad \text { for all } s \in[0,1]
$$


with

$$
a(s)=\frac{1}{(2-s)^{M-1} \mathbf{E}\left[s^{A_{M-1}}\right]},
$$

and

$$
b(s)=1-\frac{1}{(2-s)^{M-1} \mathbf{E}\left[s^{A_{M-1}}\right]}+\sum_{k=0}^{M-2} G^{(k)}(0)\left(\frac{\mathbf{E}\left[s^{A_{k}}\right]}{(2-s)^{M-1} \mathbf{E}\left[s^{A_{M-1}}\right]}-\frac{1}{(2-s)^{k}}\right) .
$$

Proof. The law of $Z_{\infty}$ is a stationary distribution for the Markov chain $Z$, therefore

$$
\begin{aligned}
G(s)=\mathbb{E}\left[\mathbb{E}_{Z_{\infty}}\left[s^{Z_{1}}\right]\right] & =\sum_{k=0}^{\infty} \mathbb{P}\left\{Z_{\infty}=k\right\} \mathbb{E}_{k}\left[s^{Z_{1}}\right] \\
& =\sum_{k=0}^{M-2} \mathbb{P}\left\{Z_{\infty}=k\right\} \mathbb{E}_{k}\left[s^{Z_{1}}\right]+\sum_{k=M-1}^{\infty} \mathbb{P}\left\{Z_{\infty}=k\right\} \mathbb{E}_{k}\left[s^{Z_{1}}\right] .
\end{aligned}
$$

By definition of $Z$, for $0 \leq k \leq M-2, Z_{1}$ under $\mathbb{P}_{k}$ has the same law as $A_{k}$ under $\mathbf{P}$. For $k \geq M-1, Z_{1}$ under $\mathbb{P}_{k}$ has the same law as $A_{M-1}+\xi_{1}+\ldots+\xi_{k-M+1}$ where $\left(\xi_{i}\right)_{i \geq 1}$ is a sequence of i.i.d. random variables independent of $A_{M-1}$ and with geometric distribution with parameter $\frac{1}{2}$. Thus,

$$
\begin{aligned}
G(s) & =\sum_{k=0}^{M-2} \mathbb{P}\left\{Z_{\infty}=k\right\} \mathbf{E}\left[s^{A_{k}}\right]+\sum_{k=M-1}^{\infty} \mathbb{P}\left\{Z_{\infty}=k\right\} \mathbf{E}\left[s^{A_{M-1}+\xi_{1}+\ldots+\xi_{k+1-M}}\right] \\
& =\sum_{k=0}^{M-2} \mathbb{P}\left\{Z_{\infty}=k\right\} \mathbf{E}\left[s^{A_{k}}\right]+\frac{\mathbf{E}\left[s^{A_{M-1}}\right]}{\mathbf{E}\left[s^{\xi}\right]^{M-1}} \sum_{k=M-1}^{\infty} \mathbb{P}\left\{Z_{\infty}=k\right\} \mathbf{E}\left[s^{\xi}\right]^{k} \\
& =\sum_{k=0}^{M-2} \mathbb{P}\left\{Z_{\infty}=k\right\}\left(\mathbf{E}\left[s^{A_{k}}\right]-\mathbf{E}\left[s^{A_{M-1}}\right] \mathbf{E}\left[s^{\xi}\right]^{k+1-M}\right)+\frac{\mathbf{E}\left[s^{A_{M-1}}\right]}{\mathbf{E}\left[s^{\xi}\right]^{M-1}} G\left(\mathbf{E}\left[s^{\xi}\right]\right) .
\end{aligned}
$$

Since $\mathbf{E}\left[s^{\xi}\right]=\frac{1}{2-s}$, and $\mathbb{P}\left\{Z_{\infty}=k\right\}=G^{k}(0)$, we get

$G(s)=\sum_{k=0}^{M-2} G^{k}(0)\left(\mathbf{E}\left[s^{A_{k}}\right]-\mathbf{E}\left[s^{A_{M-1}}\right](2-s)^{M-1-k}\right)+\mathbf{E}\left[s^{A_{M-1}}\right](2-s)^{M-1} G\left(\frac{1}{2-s}\right)$,

from which we deduce that $G$ solves (17). Furthermore, the uniqueness of the solution of this equation amongst the class of probability generating function is a direct consequence of the uniqueness of the stationary law for the irreducible Markov chain $Z$.

Given two functions $f$ and $g$, we use the classical notation $f(x)=\mathcal{O}(g(x))$ in the neighbourhood of zero if $|f(x)| \leq C|g(x)|$ for some constant $C$ and all $|x|$ small enough.

Lemma 3.5. The functions $a$ and $b$ of Lemma 3.4 are analytic on $(0,2)$. In particular, they admit a Taylor expansion of any order near point 1 and, as $x$ goes to 0 :

$$
\begin{aligned}
a(1-x) & =1-\alpha x+\mathcal{O}\left(x^{2}\right), \\
b(1-x) & =\mathcal{O}(x) .
\end{aligned}
$$


Proof. Recall the definitions of the random variables $A_{k}$ given in Section 2. Since a geometric random variable with parameter $\frac{1}{2}$ admits exponential moments of order strictly smaller than 2 , it follows that the p.g.f. $s \mapsto \mathbf{E}\left[s^{A_{k}}\right]$ are strictly positive and analytic on $(0,2)$. From the explicit form of the functions $a$ and $b$ given in the previous lemma, we conclude that these two functions are indeed analytic on $(0,2)$. A Taylor expansion of $a$ near 1 gives

$$
a(1-x)=1-\left(M-1-\mathbf{E}\left[A_{M-1}\right]\right) x+\mathcal{O}\left(x^{2}\right)=1-\alpha x+\mathcal{O}\left(x^{2}\right),
$$

where we used Lemma 3.3 for the last equality. Since $G$ is a p.g.f. we have $G(1)=1$ which, in view of (17), yields $b(1)=0$ and therefore $b(1-x)=\mathcal{O}(x)$.

The following proposition relies on a careful study of equation (17) and is the key to the proof of Theorem 1.1.

Proposition 3.6. Recall that

$$
\alpha=\sum_{i=1}^{M}\left(2 p_{i}-1\right)-1>0 .
$$

The p.g.f. $G$ of $Z_{\infty}$ is such that, as $x$ goes to 0 :

- if $0<\alpha<1$, then $1-G(1-x) \sim c_{1} x^{\alpha}$, for some constant $c_{1}>0$.

In particular $\mathbf{E}\left[Z_{\infty}\right]=+\infty$.

- if $\alpha=1$, then $1-G(1-x) \sim c_{2} x|\ln x|$, for some constant $c_{2}>0$.

In particular $\mathbf{E}\left[Z_{\infty}\right]=+\infty$.

- if $\alpha>1$, then $1-G(1-x)=c_{3} x+\mathcal{O}\left(x^{2 \wedge \alpha}\right)$ for some constant $c_{3}>0$.

In particular $\mathbf{E}\left[Z_{\infty}\right]<+\infty$.

Proof. Since $G$ is a p.g.f, it is completely monotonic and we just need to prove the proposition along the sequence $x=\frac{1}{n}$ with $n \in \mathbb{N}^{*}$. Making use of Lemma 3.4 with $s=1-\frac{1}{n}$, we get, for all $n \geq 1$

$$
1-G\left(1-\frac{1}{n+1}\right)=a\left(1-\frac{1}{n}\right)\left(1-G\left(1-\frac{1}{n}\right)\right)+b\left(1-\frac{1}{n}\right) .
$$

Let us define the sequence $\left(u_{n}\right)_{n \geq 1}$ by

$$
\left\{\begin{array}{l}
u_{1}=1-G(0)=1-\mathbf{P}\left(Z_{\infty}=0\right)>0 \\
u_{n}=\frac{1-G(1-1 / n)}{\prod_{i=1}^{n-1} a(1-1 / i)} \quad \text { for } n \geq 2
\end{array}\right.
$$

Hence, $\left(u_{n}\right)$ is a sequence of positive numbers and satisfies the equation

$$
u_{n+1}=u_{n}+\frac{b(1-1 / n)}{\prod_{i=1}^{n} a(1-1 / i)},
$$


hence

$$
u_{n}=u_{1}+\sum_{j=1}^{n-1} \frac{b(1-1 / j)}{\prod_{i=1}^{j} a(1-1 / i)} .
$$

This equality may be rewritten

$$
1-G\left(1-\frac{1}{n}\right)=\prod_{i=1}^{n-1} a\left(1-\frac{1}{i}\right)\left(1-G(0)+\sum_{j=1}^{n-1} \frac{b(1-1 / j)}{\prod_{i=1}^{j} a(1-1 / i)}\right) .
$$

Using Lemma 3.5, we easily obtain

$$
\prod_{i=1}^{n} a\left(1-\frac{1}{i}\right)=\frac{c_{4}}{n^{\alpha}}\left(1+\mathcal{O}\left(\frac{1}{n}\right)\right), \quad \text { with } c_{4}>0 .
$$

Lemma 3.5 also states that, when $b$ is not identically 0 then there exists a unique $k \in$ $\{1,2, \ldots\}$ such that

$$
b(1-x)=D_{k} x^{k}+\mathcal{O}\left(x^{k+1}\right), \quad \text { with } D_{k} \neq 0 .
$$

If $b$ is identically 0 , we use the convention $k=+\infty$. In particular, when $k$ is finite, using (21) we deduce that

$$
\frac{b(1-1 / n)}{\prod_{i=1}^{n} a(1-1 / i)}=D_{k} c_{4}^{-1} n^{\alpha-k}+\mathcal{O}\left(n^{\alpha-k-1}\right) .
$$

Let us now suppose that $k=1$. Combining (20), (21) and (22) we find that $1-G\left(1-\frac{1}{n}\right)$ converges towards $\frac{D_{1}}{\alpha} \neq 0$ as $n$ tends to infinity but this cannot happen because $G$ is continuous at $1^{-}$with $G(1)=1$. Thus, we have shown that in fact

$$
k \geq 2 .
$$

We now consider the three cases $\alpha>1, \alpha=1, \alpha<1$ separately.

$\alpha>1$

We have three sub-cases: either $\alpha>k-1$, or $\alpha<k-1$, or $\alpha=k-1$ with $k \geq 3$.

- $\underline{\alpha>k-1}$ : Making use of (22), we have

$$
\sum_{j=1}^{n-1} \frac{b(1-1 / j)}{\prod_{i=1}^{j} a(1-1 / i)}=\frac{D_{k} c_{4}^{-1}}{\alpha-k+1} n^{\alpha-k+1}+\mathcal{O}\left(1 \vee n^{\alpha-k}\right) .
$$

By (20) and (21), we deduce that

$$
1-G\left(1-\frac{1}{n}\right)=\frac{D_{k}}{(\alpha-k+1) n^{k-1}}+\mathcal{O}\left(\frac{1}{n^{k \wedge \alpha}}\right) .
$$

If $k$ was strictly larger that 2 , we would have

$$
\lim _{n \rightarrow \infty} n(1-G(1-1 / n))=0
$$


and therefore $G^{\prime}(1)=\mathbb{E}\left[Z_{\infty}\right]=0$ which cannot be true because $Z$ is a positive random variable which is not equal to zero almost surely. Thus $k$ must be equal to 2 and

$$
1-G\left(1-\frac{1}{n}\right)=\frac{D_{2}}{(\alpha-1) n}+\mathcal{O}\left(\frac{1}{n^{2 \wedge \alpha}}\right) .
$$

- $\alpha<k-1$ : We prove that this case never happens. Indeed, in view of (22) we find that, for any $\varepsilon \in(0, k-1-\alpha)$

$$
\frac{b(1-1 / n)}{\prod_{i=1}^{n} a(1-1 / i)}=\mathcal{O}\left(\frac{1}{n^{1+\varepsilon}}\right)
$$

(this result also trivially holds when $k=\infty$ ), thus

$$
\sum_{j=1}^{\infty} \frac{b(1-1 / j)}{\prod_{i=1}^{j} a(1-1 / i)}<\infty
$$

Combining this with (20) and (21) we see that

$$
1-G\left(1-\frac{1}{n}\right)=\mathcal{O}\left(\frac{1}{n^{\alpha}}\right) .
$$

Since $\alpha>1$, just as in the previous case, this implies that $\mathbb{E}\left[Z_{\infty}\right]=0$ which is absurd.

- $\underline{\alpha=k-1 \text { and } k \geq 3}$ : Again, we prove that this case is empty. Using (22), we now get

$$
\frac{b(1-1 / n)}{\prod_{i=1}^{n} a(1-1 / i)} \sim \frac{D_{k} c_{4}^{-1}}{n} .
$$

And, by (20) and (21), we conclude that

$$
1-G\left(1-\frac{1}{n}\right) \sim D_{k} \frac{\ln n}{n^{k-1}} .
$$

Since $k \geq 3$, we obtain $\mathbb{E}\left[Z_{\infty}\right]=0$ which is unacceptable.

Thus, we have completed the proof of the proposition when $\alpha>1$ and we proved by the way that $k$ must be equal to 2 .

$\alpha=1$

We first prove, just as in the previous cases, that $k=2$. Let us suppose that $k \geq 3$. In view of Lemma 3.5, for any $l \geq 3$, we can write the Taylor expansion of $b$ of order $l$ near 1 in the form

$$
b(1-x)=D_{3} x^{3}+\ldots+D_{l} x^{l}+\mathcal{O}\left(x^{l+1}\right)
$$

where $D_{i} \in \mathbb{R}$ for $i \in\{3,4, \ldots, l\}$. Similarly,

$$
a(1-x)=1-x+a_{2} x^{2}+\ldots+a_{l} x^{l}+\mathcal{O}\left(x^{l+1}\right),
$$


from which we deduce that, as $n$ goes to infinity

$$
\prod_{i=1}^{n} a\left(1-\frac{1}{i}\right)=\frac{a_{1}^{\prime}}{n}+\frac{a_{2}^{\prime}}{n^{2}}+\ldots+\frac{a_{l}^{\prime}}{n^{l}}+\mathcal{O}\left(\frac{1}{n^{l+1}}\right) \quad \text { with } a_{1}^{\prime}>0 .
$$

From (25) and (26) we also deduce that

$$
\frac{b(1-1 / n)}{\prod_{i=1}^{n} a(1-1 / i)}=\frac{d_{2}^{\prime}}{n^{2}}+\ldots+\frac{d_{l-1}^{\prime}}{n^{l-1}}+\mathcal{O}\left(\frac{1}{n^{l}}\right) .
$$

Thus,

$$
\sum_{j=1}^{n-1} \frac{b(1-1 / j)}{\prod_{i=1}^{j} a(1-1 / i)}=g_{0}+\frac{g_{1}}{n}+\frac{g_{2}}{n^{2}}+\ldots+\frac{g_{l-2}}{n^{l-2}}+\mathcal{O}\left(\frac{1}{n^{l-1}}\right) .
$$

Therefore, in view of (20), (26) and (27), we get

$$
\begin{aligned}
1-G\left(1-\frac{1}{n}\right) & =\prod_{i=1}^{n-1} a\left(1-\frac{1}{i}\right)\left(1-G(0)+\sum_{j=1}^{n-1} \frac{b(1-1 / j)}{\prod_{i=1}^{j} a(1-1 / i)}\right) \\
& =\frac{\lambda_{1}}{n}+\frac{\lambda_{2}}{n^{2}}+\ldots+\frac{\lambda_{l-1}}{n^{l-1}}+\mathcal{O}\left(\frac{1}{n^{l}}\right) .
\end{aligned}
$$

Comparing with the Taylor expansion of the p.g.f. $G$, we conclude that $\mathbf{E}\left(Z_{\infty}^{l-1}\right)<\infty$ for all $l$ which contradicts Proposition 3.1. Thus, $k=2$ and (22) yields

$$
\frac{b(1-1 / n)}{\prod_{i=1}^{n} a(1-1 / i)} \sim \frac{D_{2} c_{4}^{-1}}{n} \quad \text { with } D_{2} \neq 0 .
$$

And, by (201) and (21), we conclude that

$$
1-G\left(1-\frac{1}{n}\right) \sim D_{2} \frac{\ln n}{n}
$$

and therefore

$$
\mathbb{E}\left[Z_{\infty}\right]=+\infty
$$

$\alpha<1$

Since $k \geq 2$, the relation (22) yields

$$
\sum_{j=1}^{\infty} \frac{b(1-1 / j)}{\prod_{i=1}^{j} a(1-1 / i)}<\infty
$$

(of course, this is trivially true when $k=\infty$ ). Thus, the sequence $\left(u_{n}\right)$ defined by (19) converges to a constant $c_{5} \geq 0$. Suppose first that $c_{5}=0$. In this case, $k$ cannot be infinite (because when $k=\infty$, the sequence $\left(u_{n}\right)$ is constant and then $c_{5}=u_{1}>0$ ). From (22) we deduce that

$$
u_{n}=-\sum_{j=n}^{\infty} \frac{b(1-1 / j)}{\prod_{i=1}^{j} a(1-1 / i)} \sim \frac{D_{k}}{(k-\alpha-1) c_{4} n^{k-\alpha-1}}
$$


therefore, with the help of (21) we get that

$$
1-G\left(1-\frac{1}{n}\right)=u_{n} \prod_{i=1}^{n-1} a\left(1-\frac{1}{i}\right) \sim \frac{D_{k}}{(k-\alpha-1) n^{k-1}} .
$$

Since $k \geq 2$, this implies that $n(1-G(1-1 / n))$ converges to a finite constant and so $\mathbf{E}\left[Z_{\infty}\right]<\infty$. We already notice that this implies a strict positive speed for the cookie random walk in the associated cookie environment $(M, \bar{p})$. But (by possibly extending the value of $M)$ we can always construct a cookie environment $(M, \bar{q})$ such that $\bar{p} \leq \bar{q}$ and $\alpha(\bar{q})=1$. In view of $(28)$, the associated cookie random walk has a zero speed and this contradicts a monotonicity result of Zerner (c.f. Theorem 17 of [10]). Therefore $c_{5}$ cannot be 0 and by (19) and (21), we get that

$$
1-G\left(1-\frac{1}{n}\right)=u_{n} \prod_{i=1}^{n-1} a\left(1-\frac{1}{i}\right) \sim \frac{c_{5} c_{4}}{n^{\alpha}} .
$$

As we already noticed, Theorem 1.1 is now a direct consequence of the last proposition and Corollary 2.3. We also proved that, when the speed is strictly positive, its value is given by the formula

$$
v=\frac{\alpha-1}{\alpha-1+2 D_{2}} \quad \text { where } 2 D_{2}=b^{\prime \prime}(1)>0 .
$$

Remark 3.7. In the transient case and when the limiting speed is zero, Proposition $[3.6$ gives with the help of a classical Abelian/Tauberian Theorem the asymptotic of the distribution tail of $Z_{\infty}$ i.e. the distribution tail of the total number of jumps from 0 to -1 :

$$
\mathbb{P}\left\{Z_{\infty}>n\right\} \underset{n \rightarrow \infty}{\sim} \begin{cases}\frac{c_{6}}{n^{\alpha}} & \text { if } 0<\alpha<1 \\ \frac{c_{7} \ln n}{n} & \text { if } \alpha=1 .\end{cases}
$$

The functional equation given in Lemma 3.4 for the p.g.f. of $Z_{\infty}$ also gives a similar equation for the total number of returns $R$ to the origin for the cookie random walk. Indeed, recall that $U_{0}^{n}$ (resp. $U_{1}^{n}$ ) stands for the respective total number of jumps from 0 to -1 (resp. from 1 to 0 ) before reaching level $n$. Thus, the total number of returns to the origin before reaching level $n$ is $U_{0}^{n}+U_{1}^{n}$ which, under $\mathbf{P}$ has the same distribution as $Z_{n}+Z_{n-1}$ under $\mathbb{P}$. Therefore, we can express the p.g.f. $H$ of the random variable $R$ in term of $G$ :

$$
\begin{aligned}
H(s) & =\mathbb{E}\left[s^{Z_{\infty}} \mathbb{E}_{Z_{\infty}}\left[s^{Z_{\infty}}\right]\right] \\
& =\frac{1}{a(s)} G\left(\frac{s}{2-s}\right)+\sum_{k=0}^{M-2} G^{(k)}(0) s^{k}\left(\mathbb{E}\left[s^{A_{k}}\right]-\frac{1}{a(s)(2-s)^{k}}\right) .
\end{aligned}
$$

In particular, Proposition 3.6 also holds for $H$ and the tail distribution of the total number of returns to the origin when $\alpha \leq 1$ has the same form as in (29). 
Remark 3.8. In the particular case $M=2$ (there are at most 2 cookies per site), the only unknown in the definition of the function $b$ is $G(0)$. Since we know that $b^{\prime}(1)=0$ (c.f. the beginning of the proof of Proposition 3.6) we can therefore explicitly calculate $G(0)$, that is the probability that the cookie random walk never jumps from 0 to 1 which is also the probability that the cookie random walk never hits -1. According to the previous remark, we can also calculate the probability that the cookie random walk never returns to 0. Hence, we recover Theorem 18 of [10] in the case of a deterministic cookie environment.

\section{Continuity of the speed and differentiability at the critical point}

The aim of this section is to prove Theorem 1.2, Recall that

$$
v(M, \bar{p})= \begin{cases}0 & \text { if } \alpha(M, p) \leq 1 \\ \frac{\alpha-1}{\alpha-1+b^{\prime \prime}(1)} & \text { if } \alpha(M, p)>1\end{cases}
$$

where $b^{\prime \prime}(1)$ stands for the second derivative at point 1 of the function $b$ defined in Lemma 3.4

$$
b(s)=1-\frac{1}{(2-s)^{M-1} \mathbf{E}\left[s^{A_{M-1}}\right]}+\sum_{k=0}^{M-2} \mathbb{P}\left\{Z_{\infty}=k\right\}\left(\frac{\mathbf{E}\left[s^{A_{k}}\right]}{(2-s)^{M-1} \mathbf{E}\left[s^{A_{M-1}}\right]}-\frac{1}{(2-s)^{k}}\right) .
$$

Furthermore, we also proved in Proposition 3.6 that, when $\alpha(M, \bar{p})=1$, then $b^{\prime \prime}(1)$ is strictly positive. Hence, in order to prove Theorem 1.2, we just need to show that $b^{\prime \prime}(1)=b_{(M, \bar{p})}^{\prime \prime}(1)$ is a continuous function of $\bar{p}$ in $\Omega_{M}^{u}$. It is also clear from the definition of the random variables $A_{k}$ that the functions

$$
\bar{p} \rightarrow\left(\mathbf{E}_{(M, \bar{p})}\left[s^{A_{k}}\right]\right)^{(i)}(1) \quad\left(\text { i.e. the } i^{\text {th }} \text { derivative at point } 1\right)
$$

are continuous in $\bar{p}$ in $\Omega_{M}^{u}$ for all $k \geq 0$ and all $i \geq 0$ (it is a rational function in $p_{1}, \ldots, p_{M}$ ). Therefore, it simply remains to prove that, for any $k \geq 0$, the function

$$
\bar{p} \rightarrow \mathbb{P}_{(M, \bar{p})}\left\{Z_{\infty}=k\right\}
$$

is continuous in $\Omega_{M}^{u}$. The following lemma is based on the monotonicity of the hitting times of a cookie random walk with respect to the environment.

Lemma 4.1. Let $(M, \bar{p})$ be a cookie environment such that $\alpha(M, \bar{p})>0$. Then there exist $\varepsilon>0$ and $f: \mathbb{N} \mapsto \mathbb{R}_{+}$with $\lim _{n \rightarrow+\infty} f(n)=0$ such that

$$
\forall \bar{q} \in B(\bar{p}, \varepsilon), \forall j \in \mathbb{N}, \forall n \in \mathbb{N},\left|\mathbb{P}_{(M, \bar{q})}\left\{Z_{\infty}=j\right\}-\mathbb{P}_{(M, \bar{q})}\left\{Z_{n}=j\right\}\right| \leq f(n),
$$

where

$$
B(\bar{p}, \varepsilon)=\left\{\bar{q}=\left(q_{1}, \ldots, q_{M}\right), \frac{1}{2} \leq q_{i}<1, \alpha(M, \bar{q})>0 \text { and } \sum_{i=1}^{\infty}\left|p_{i}-q_{i}\right| \leq \varepsilon\right\} .
$$


Proof. Let us fix $(M, \bar{p})$ with $\alpha(M, \bar{p})>0$. For $\varepsilon>0$, define the vector $\bar{p}^{\varepsilon}=\left(p_{1}^{\varepsilon}, \ldots, p_{M}^{\varepsilon}\right)$ by $p_{i}^{\varepsilon}=\max \left(\frac{1}{2}, p_{i}-\varepsilon\right)$. We can choose $\varepsilon>0$ such that $\alpha\left(M, \bar{p}^{\varepsilon}\right)>0$. Then, for all $\bar{q} \in B(\bar{p}, \varepsilon)$, we have

$$
\bar{p}^{\varepsilon} \leq \bar{q}
$$

(where $\leq$ denotes the canonical partial order on $\mathbb{R}^{M}$ ). Let now pick $\bar{q} \in B(\bar{p}, \varepsilon), j \in \mathbb{N}$ and $n \in \mathbb{N}$. Recall that $U_{0}^{\infty}$ denotes the total number of jump of the cookie random walk from 0 to -1 and

$$
\mathbb{P}_{(M, \bar{q})}\left\{Z_{\infty}=j\right\}=\mathbf{P}_{(M, \bar{q})}\left\{U_{0}^{\infty}=j\right\}=\mathbf{P}_{(M, \bar{q})}\{X \text { jumps } j \text { times from } 0 \text { to }-1\},
$$

and

$$
\begin{aligned}
\mathbb{P}_{(M, \bar{q})}\left\{Z_{n}=j\right\}=\mathbf{P}_{(M, \bar{q})}\left\{U_{0}^{n}=j\right\} & \\
& =\mathbf{P}_{(M, \bar{q})}\{X \text { jumps } j \text { times from } 0 \text { to }-1 \text { before reaching } n\} .
\end{aligned}
$$

Hence

$$
\begin{aligned}
\left|\mathbb{P}_{(M, \bar{q})}\left\{Z_{\infty}=j\right\}-\mathbb{P}_{(M, \bar{q})}\left\{Z_{n}=j\right\}\right| & =\left|\mathbf{P}_{(M, \bar{q})}\left\{U_{0}^{\infty}=j\right\}-\mathbf{P}_{(M, \bar{q})}\left\{U_{0}^{n}=j\right\}\right| \\
& \leq \mathbf{P}_{(M, \bar{q})}\left\{U_{0}^{n} \neq U_{0}^{\infty}\right\} \\
& =\mathbf{P}_{(M, \bar{q})}\{A\},
\end{aligned}
$$

where $A$ is the event " $X$ visits -1 at least once after reaching level $n$ ". Recall the notation $\omega=\omega(i, x)_{i \geq 1, x \in \mathbb{Z}}$ for a general cookie environment given in the introduction. Let now $\omega_{X, n}$ denote the (random) cookie-environment obtained when the cookie random walk $X$ hits level $n$ for the first time and shifted by $n$, i.e. for all $x \in \mathbb{Z}$ and $i \geq 1$, if the initial cookie environment is $\omega$, then

$$
\omega_{X, n}(i, x)=\omega(j, x+n) \quad \text { where } j=i+\sharp\left\{0 \leq k<T_{n}, X_{k}=x+n\right\} .
$$

With this notation we have

$$
\mathbf{P}_{(M, \bar{q})}\{A\}=\mathbf{E}_{(M, \bar{q})}\left[\mathbf{P}_{\omega_{X, n}}\{X \text { visits }-(n+1) \text { at least once }\}\right] .
$$

Besides, $X$ has not eaten any cookie at the sites $x \geq n$ before time $T_{n}$. Thus, the environment $\omega_{X, n}$ satisfies $\mathbf{P}_{(M, \bar{q})}$-almost surely

$\omega_{X, n}(i, x)=q_{i}, \quad$ for all $x \geq 0$ and $i \geq 1$ (with the convention $q_{i}=\frac{1}{2}$ for $i>M$ ).

Hence, in view of (30), the random cookie environment $\omega_{X, n}$ is $\mathbf{P}_{(M, \bar{q})^{-a l m o s t}}$ surely larger (for the canonical partial order) than the deterministic environment $\omega_{\bar{p}^{\varepsilon}}$ defined by

$$
\left\{\begin{array}{l}
\omega_{\bar{p}^{\varepsilon}}(i, x)=\frac{1}{2}, \quad \text { for all } x<0 \text { and } i \geq 1, \\
\left.\omega_{\bar{p}^{\varepsilon}}(i, x)=p_{i}^{\varepsilon}, \quad \text { for all } x \geq 0 \text { and } i \geq 1 \text { (with the convention } p_{i}^{\varepsilon}=\frac{1}{2} \text { for } i \geq M\right) .
\end{array}\right.
$$

Thus, Lemma 15 of [10] yields

$$
\begin{aligned}
& \mathbf{P}_{\omega_{X, n}}\{X \text { visits }-(n+1) \text { at least once }\} \\
& \quad \leq \mathbf{P}_{\omega_{\bar{p}^{\varepsilon}}}\{X \text { visits }-(n+1) \text { at least once }\} \quad \mathbf{P}_{(M, \bar{q})}-\text { a.s. }
\end{aligned}
$$


In view of (31) we deduce that

$$
\left|\mathbb{P}_{(M, \bar{q})}\left\{Z_{\infty}=j\right\}-\mathbb{P}_{(M, \bar{q})}\left\{Z_{n}=j\right\}\right| \leq f(n),
$$

where $f(n)=\mathbf{P}_{\omega_{\bar{p}^{\varepsilon}}}\{X$ visits $-(n+1)$ at least once $\}$ does not depend of $\bar{q}$. It remains to prove that $f(n)$ tends to 0 as $n$ goes to infinity. Let us first notice that

$$
\mathbf{P}_{\omega_{\bar{p}^{\varepsilon}}}\left\{\forall n \geq 0, X_{n} \geq 0\right\}=\mathbf{P}_{\left(M, \bar{p}^{\varepsilon}\right)}\left\{\forall n \geq 0, X_{n} \geq 0\right\}
$$

since these probabilities depend only on the environments on the half line $[0,+\infty)$. Recall also that the cookie random walk in the environment $\left(M, \bar{p}^{\varepsilon}\right)$ is transient (we have chosen $\varepsilon$ such that $\left.\alpha\left(M, \bar{p}^{\varepsilon}\right)>0\right)$, thus

$$
\mathbf{P}_{\left(M, \bar{p}^{\varepsilon}\right)}\left\{\forall n \geq 0, X_{n} \geq 0\right\}=\mathbf{P}_{\left(M, \bar{p}^{\varepsilon}\right)}\left\{U_{0}^{\infty}=0\right\}=\mathbb{P}_{\left(M, \bar{p}^{\varepsilon}\right)}\left\{Z_{\infty}=0\right\}>0 .
$$

Hence

$$
\mathbf{P}_{\omega_{\bar{p}^{\varepsilon}}}\left\{\forall n \geq 0, X_{n} \geq 0\right\}>0,
$$

which implies

$$
\mathbf{P}_{\omega_{\bar{p}} \varepsilon}\left\{X_{n}=0 \text { infinitely often }\right\}<1,
$$

and a $0-1$ law (c.f. Proposition 5 of [10]) yields

$$
\mathbf{P}_{\omega_{\bar{p} \varepsilon}^{\varepsilon}}\left\{X_{n}=0 \text { infinitely often }\right\}=\mathbf{P}_{\omega_{\bar{p} \varepsilon}}\left\{X_{n} \leq 0 \text { infinitely often }\right\}=0 .
$$

Therefore, $\lim _{n \rightarrow \infty} f(n)=0$.

Recall that the transition probabilities of the Markov chain $Z$ are given by the law of the random variables $A_{k}$ :

$$
\mathbb{P}_{(M, \bar{p})}\left\{Z_{n+1}=j \mid Z_{n}=i\right\}=\mathbf{P}_{(M, \bar{p})}\left\{A_{i}=j\right\} .
$$

It is therefore clear that for each fixed $n$ and each $k$, the function $\bar{p} \rightarrow \mathbb{P}_{(M, \bar{p})}\left\{Z_{n}=k\right\}$ is continuous in $\bar{p}$ in $\Omega_{M}^{u}$. In view of the previous lemma, we conclude that for each $k$ the function $\bar{p} \rightarrow \mathbb{P}_{(M, \bar{p})}\left\{Z_{\infty}=k\right\}$ is also continuous in $\bar{p}$ in $\Omega_{M}^{u}$ and this completes the proof of Theorem 1.2 .

Acknowledgments. The authors would like to thank Yueyun Hu for all his precious advices.

\section{References}

[1] T. Antal and S. Redner. The excited random walk in one dimension. J. Phys. A, 38(12):2555-2577, 2005.

[2] I. Benjamini and D. B. Wilson. Excited random walk. Electron. Comm. Probab., 8:86-92 (electronic), 2003. Available via http://www.math. washington. edu/ ejpecp/EcpVol8/paper9.abs.html.

[3] B. Davis. Brownian motion and random walk perturbed at extrema. Probab. Theory Related Fields, 113(4):501-518, 1999. 
[4] S. V. Kaverin. Refinement of limit theorems for critical branching processes with emigration. Teor. Veroyatnost. i Primenen., 35(3):570-575, 1990. Translated in Theory Probab. Appl. 35 (1990), no. 3, 574-580 (1991).

[5] H. Kesten, M. V. Kozlov, and F. Spitzer. A limit law for random walk in a random environment. Compositio Math., 30:145-168, 1975.

[6] G. Kozma. Excited random walk in three dimensions has positive speed, 2003. Preprint, available via http://arxiv.org/abs/math.PR/0310305.

[7] G. Kozma. Excited random walk in two dimensions has linear speed, 2005. Preprint, available via http://arxiv.org/abs/math.PR/0512535.

[8] T. Mountford, L. P. R. Pimentel, and G. Valle. On the speed of the onedimensional excited random walk in the transient regime, 2006. Preprint, available via http://arxiv.org/abs/math.PR/0602041.

[9] V. A. Vatutin and A. M. Zubkov. Branching processes. II. J. Soviet Math., 67(6):3407-3485, 1993. Probability theory and mathematical statistics, 1.

[10] M. P. W. Zerner. Multi-excited random walks on integers. Probab. Theory Related Fields, 133(1):98-122, 2005.

[11] M. P. W. Zerner. Recurrence and transience of excited random walks on $\mathbb{Z}^{d}$ and strips. Electron. Comm. Probab., 11:118-128 (electronic), 2006. Available via http://www.math.washington.edu/ ejpecp/EcpVol11/paper12.abs.html. 